NBER WORKING PAPER SERIES

\title{
IMPROVING GDP MEASUREMENT: A FORECAST COMBINATION PERSPECTIVE
}

\author{
S. Boragan Aruoba \\ Francis X. Diebold \\ Jeremy Nalewaik \\ Frank Schorfheide \\ Dongho Song \\ Working Paper 17421 \\ http://www.nber.org/papers/w17421
NATIONAL BUREAU OF ECONOMIC RESEARCH
1050 Massachusetts Avenue
Cambridge, MA 02138
September 2011

The views expressed herein are those of the authors and do not necessarily reflect the views of the National Bureau of Economic Research. For research support we thank the National Science Foundation and the Real-Time Data Research Center at the Federal Reserve Bank of Philadelphia.

NBER working papers are circulated for discussion and comment purposes. They have not been peerreviewed or been subject to the review by the NBER Board of Directors that accompanies official NBER publications.

(C) 2011 by S. Boragan Aruoba, Francis X. Diebold, Jeremy Nalewaik, Frank Schorfheide, and Dongho Song. All rights reserved. Short sections of text, not to exceed two paragraphs, may be quoted without explicit permission provided that full credit, including $\odot$ notice, is given to the source. 
Improving GDP Measurement: A Forecast Combination Perspective

S. Boragan Aruoba, Francis X. Diebold, Jeremy Nalewaik, Frank Schorfheide, and Dongho

Song

NBER Working Paper No. 17421

September 2011

JEL No. E01,E32

\title{
ABSTRACT
}

Two often-divergent U.S. GDP estimates are available, a widely-used expenditure side version, GDPE, and a much less widely-used income-side version GDPI . We propose and explore a "forecast combination" approach to combining them. We then put the theory to work, producing a superior combined estimate of GDP growth for the U.S., GDPC. We compare GDPC to GDPE and GDPI, with particular attention to behavior over the business cycle. We discuss several variations and extensions.

\author{
S. Boragan Aruoba \\ Frank Schorfheide \\ Department of Economics \\ University of Pennsylvania \\ University of Maryland \\ 3105 Tydings Hall \\ College Park, MD 20742-7211 \\ Department of Economics \\ 3718 Locust Walk \\ aruoba@econ.umd.edu \\ McNeil 525 \\ Philadelphia, PA 19104-6297 \\ and NBER \\ Francis X. Diebold \\ schorf@ssc.upenn.edu \\ Department of Economics \\ University of Pennsylvania \\ Dongho Song \\ 3718 Locust Walk \\ University of Pennsylvania \\ Philadelphia, PA 19104-6297 \\ Department of Economics \\ and NBER \\ 3718 Locust Walk \\ fdiebold@sas.upenn.edu \\ Philadelphia, PA 19104 \\ donghos@sas.upenn.edu \\ Jeremy Nalewaik \\ Federal Reserve Board \\ 20th and C, NW \\ Washington, DC 20551 \\ jeremy.j.nalewaik@frb.gov
}


"A growing number of economists say that the government should shift its approach to measuring growth. The current system emphasizes data on spending, but the bureau also collects data on income. In theory the two should match perfectly - a penny spent is a penny earned by someone else. But estimates of the two measures can diverge widely, particularly in the short term..."

[Binyamin Appelbaum, New York Times, August 16, 2011]

\section{Introduction}

GDP growth is surely the most fundamental and important concept in empirical/applied macroeconomics and business cycle monitoring, yet significant uncertainty still surrounds its estimation. Two often-divergent estimates exist for the U.S., a widely-used expenditure side version, $G D P_{E}$, and a much less-widely-used income-side version, $G D P_{I}$. Nalewaik (2010) makes clear that, at the very least, $G D P_{I}$ deserves serious attention and may even have properties in certain respects superior to those of $G D P_{E}$. That is, if forced to choose between $G D P_{E}$ and $G D P_{I}$, a surprisingly strong case exists for $G D P_{I}$.

But of course one is not forced to choose between $G D P_{E}$ and $G D P_{I}$, and a combined estimate that pools information in the two indicators $G D P_{E}$ and $G D P_{I}$ may improve on both. In this paper we propose and explore a method for constructing such a combined estimate, and we compare our new $G D P_{C}$ ("combined") series to $G D P_{E}$ and $G D P_{I}$ over many decades, with particular attention to behavior over the business cycle, emphasizing comparative behavior during turning points.

Our work is motivated by, and builds upon, four key literatures. First, we obviously build on the literature examining $G D P_{I}$ and its properties, notably Fixler and Nalewaik (2009) and Nalewaik (2010). Second, our work is related to the literature distinguishing between "forecast error" and "measurement error" data revisions, as for example in Mankiw et al. (1984), Mankiw and Shapiro (1986), Faust et al. (2005), and Aruoba (2008). In this paper we work largely in the forecast error tradition. Third, and related, we work in the tradition of the forecast combination literature begun by Bates and Granger (1969), viewing $G D P_{E}$ and $G D P_{I}$ as forecasts of GDP (actually a mix of "backcasts" and "nowcasts" in the parlance of Aruoba and Diebold (2010)). We combine those forecasts by forming optimally 
weighted averages. ${ }^{1}$ Finally, and most pleasing to us, our work is very much related to Hal White's, both in its focus on dynamic modeling and prediction and in its acknowledgment of misspecification throughout.

We proceed as follows. In section 2 we consider GDP combination under quadratic loss. This involves taking a stand on the values of certain unobservable parameters (or at least reasonable ranges for those parameters), but we argue that a "quasi-Bayesian" calibration procedure based on informed judgment is feasible, credible and robust. In section 3 we consider GDP combination under minimax loss. Interestingly, as we show, it does not require calibration. In section 4 we apply our methods to provide improved GDP estimates for the U.S. In section 5 we sketch several extensions, and we conclude in section 6 .

\section{Combination Under Quadratic Loss}

Optimal forecast combination typically requires knowledge (or, in practice, estimates) of forecast error properties such as variances and covariances. In the present context, we have two "forecasts," of true GDP, namely $G D P_{E}$ and $G D P_{I}$, but true GDP is never observed, even after the fact. Hence we never see the "forecast errors," which complicates matters significantly but not hopelessly. In particular, in this section we work under quadratic loss and show that a quasi-Bayesian calibration based on informed judgment is feasible and credible, and simultaneously, that the efficacy of GDP combination is robust to the precise weights used.

\subsection{Basic Results and Calibration}

First assume that the errors in $G D P_{E}$ and $G D P_{I}$ growth are uncorrelated. Consider the convex combination ${ }^{2}$

$$
G D P_{C}=\lambda G D P_{E}+(1-\lambda) G D P_{I}
$$

\footnotetext{
${ }^{1}$ For forecast combination surveys see Diebold and Lopez (1996) and Timmermann (2006).

${ }^{2}$ Throughout this paper, GDP, GDP $E$ and $G D P_{I}$ refer to growth rates.
} 
where $\lambda \in[0,1] .{ }^{3}$ Then the associated errors follow the same weighting,

$$
e_{C}=\lambda e_{E}+(1-\lambda) e_{I},
$$

where $e_{C}=\mathrm{GDP}-G D P_{C}, e_{E}=\mathrm{GDP}-G D P_{E}$ and $e_{I}=\mathrm{GDP}-G D P_{I}$. Assume that both $G D P_{E}$ and $G D P_{I}$ are unbiased for GDP, in which case $G D P_{C}$ is also unbiased, because the combining weights sum to unity.

Given the unbiasedness assumption, the minimum-MSE combining weights are just the minimum-variance weights. Immediately, using the assumed zero correlation between the errors,

$$
\sigma_{C}^{2}=\lambda^{2} \sigma_{E}^{2}+(1-\lambda)^{2} \sigma_{I}^{2},
$$

where $\sigma_{C}^{2}=\operatorname{var}\left(e_{C}\right), \sigma_{E}^{2}=\operatorname{var}\left(e_{E}\right)$ and $\sigma_{I}^{2}=\operatorname{var}\left(e_{I}\right)$. Minimization with respect to $\lambda$ yields the optimal combining weight,

$$
\lambda^{*}=\frac{\sigma_{I}^{2}}{\sigma_{I}^{2}+\sigma_{E}^{2}}=\frac{1}{1+\phi^{2}},
$$

where $\phi=\sigma_{E} / \sigma_{I}$.

It is interesting and important to note that in the present context of zero correlation between the errors,

$$
\operatorname{var}\left(e_{E}\right)+\operatorname{var}\left(e_{I}\right)=\operatorname{var}\left(G D P_{E}-G D P_{I}\right) .
$$

The standard deviation of $G D P_{E}$ minus $G D P_{I}$ can be trivially estimated. Thus, an expression of a view about $\phi$ is in fact implicitly an expression of a view about not only the ratio of $\operatorname{var}\left(e_{E}\right)$ and $\operatorname{var}\left(e_{I}\right)$, but about their actual values. We will use this fact (and its generalization in the case of correlated errors) in several places in what follows.

Based on our judgment regarding U.S. $G D P_{E}$ and $G D P_{I}$ data, which we will subsequently discuss in detail in section 2.2, we believe that a reasonable range for $\phi$ is $\phi \in[.75,1.45]$, with midpoint 1.10. ${ }^{4}$ One could think of this as a quasi-Bayesian statement that prior beliefs regarding $\phi$ are centered at 1.10, with a ninety percent prior credible interval of $[.75,1.45]$.

\footnotetext{
${ }^{3}$ Strictly speaking, we need not even impose $\lambda \in[0,1]$, but $\lambda \notin[0,1]$ would be highly non-standard for two valuable and sophisticated GDP estimates such as $G D P_{E}$ and $G D P_{I}$. Moreover, as we shall see subsequently, multiple perspectives suggest that for our application the interesting range of $\lambda$ is well in the interior of the unit interval.

${ }^{4}$ Invoking equation (3), we see that the midpoint 1.10 corresponds to $\sigma_{I}=1.30$ and $\sigma_{E}=1.43$, given our estimate of $\operatorname{std}\left(G D P_{E}-G D P_{I}\right)=1.93$ percent using data 1947Q2-2009Q3.
} 


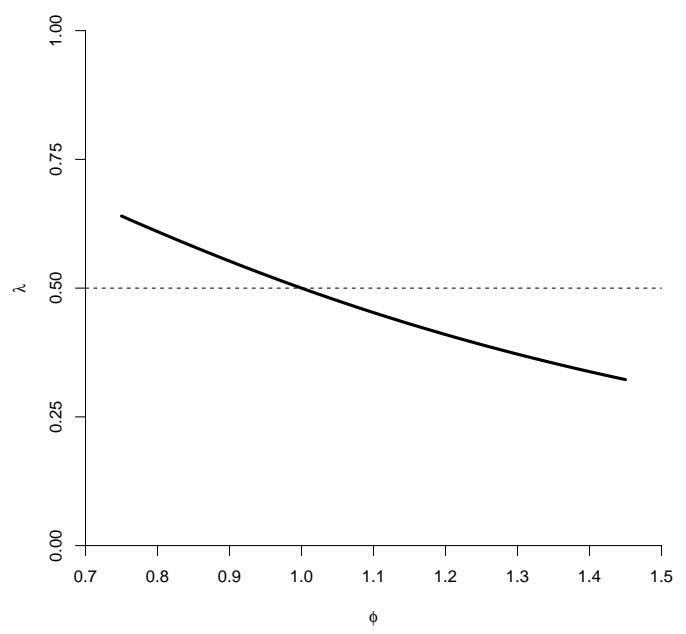

Figure 1: $\lambda^{*}$ vs. $\phi . \lambda^{*}$ constructed assuming uncorrelated errors. The horizontal line for visual reference is at $\lambda^{*}=.5$. See text for details.

In Figure 1 we graph $\lambda^{*}$ as a function of $\phi$, for $\phi \in[.75,1.45] . \lambda^{*}$ is of course decreasing in $\phi$, but interestingly, it is only mildly sensitive to $\phi$. Indeed, for our range of $\phi$ values, the optimal combining weight remains close to 0.5 , varying from roughly 0.65 to 0.30 . At the midpoint $\phi=1.10$, we have $\lambda^{*}=0.45$.

It is instructive to compare the error variance of combined GDP, $\sigma_{C}^{2}$, to $\sigma_{E}^{2}$ for a range of $\lambda$ values (including $\lambda=\lambda^{*}, \lambda=0$, and $\left.\lambda=1\right) .{ }^{5}$ From (1) we have:

$$
\frac{\sigma_{C}^{2}}{\sigma_{E}^{2}}=\lambda^{2}+\frac{(1-\lambda)^{2}}{\phi^{2}}
$$

In Figure 2 we graph $\sigma_{C}^{2} / \sigma_{E}^{2}$ for $\lambda \in[0,1]$ with $\phi=1.1$. Obviously the maximum variance reduction is obtained using $\lambda^{*}=0.45$, but even for non-optimal $\lambda$, such as simple equalweight combination $(\lambda=0.5)$, we achieve substantial variance reduction relative to using $G D P_{E}$ alone. Indeed a key result is that for all $\lambda$ (except those very close to 1 , of course) we achieve substantial variance reduction.

Now consider the more general and empirically-relevant case of correlated errors. Under

\footnotetext{
${ }^{5}$ We choose to examine $\sigma_{C}^{2}$ relative to $\sigma_{E}^{2}$, rather than to $\sigma_{I}^{2}$, because $G D P_{E}$ is the "standard" GDP estimate used in practice almost universally. A graph of $\sigma_{C}^{2} / \sigma_{I}^{2}$ would be qualitatively identical, but the drop below 1.0 would be less extreme.
} 


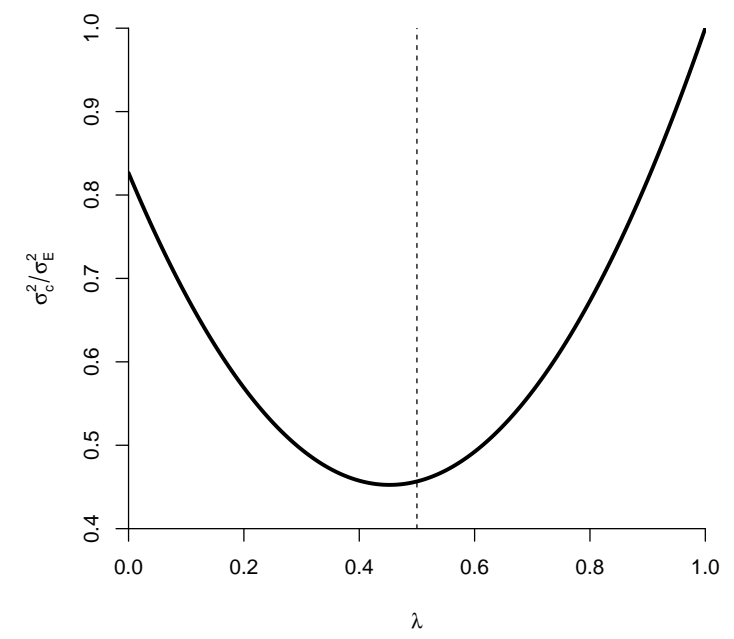

Figure 2: $\sigma_{C}^{2} / \sigma_{E}^{2}$ for $\lambda \in[0,1]$. We assume $\phi=1.1$ and uncorrelated errors. See text for details.

the same conditions as earlier,

$$
\sigma_{c}^{2}=\lambda^{2} \sigma_{E}^{2}+(1-\lambda)^{2} \sigma_{I}^{2}+2 \lambda(1-\lambda) \sigma_{E I}
$$

SO

$$
\begin{aligned}
\lambda^{*} & =\frac{\sigma_{I}^{2}-\sigma_{E I}}{\sigma_{I}^{2}+\sigma_{E}^{2}-2 \sigma_{E I}} \\
& =\frac{1-\phi \rho}{1+\phi^{2}-2 \phi \rho},
\end{aligned}
$$

where $\sigma_{E I}=\operatorname{cov}\left(e_{E}, e_{I}\right)$ and $\rho=\operatorname{corr}\left(e_{E}, e_{I}\right)$.

It is noteworthy that - in parallel to the uncorrelated-error case in which beliefs about $\phi$ map one-for-one into beliefs about $\sigma_{E}$ and $\sigma_{I}$ - beliefs about $\phi$ and $\rho$ now similarly map one-for-one into beliefs about $\sigma_{E}$ and $\sigma_{I}$. Our definitions of $\sigma_{E}^{2}$ and $\sigma_{I}^{2}$ imply that

$$
\sigma_{j}^{2}=\operatorname{var}\left[G D P_{j}\right]-2 \operatorname{cov}\left[G D P_{j}, G D P\right]+\operatorname{var}[G D P], \quad j \in\{E, I\}
$$

Moreover, the covariance between the $G D P_{E}$ and $G D P_{I}$ errors can be expressed as

$$
\sigma_{E I}=\operatorname{cov}\left[G D P_{E}, G D P_{I}\right]-\operatorname{cov}\left[G D P_{E}, G D P\right]-\operatorname{cov}\left[G D P_{I}, G D P\right]+\operatorname{var}[G D P]
$$



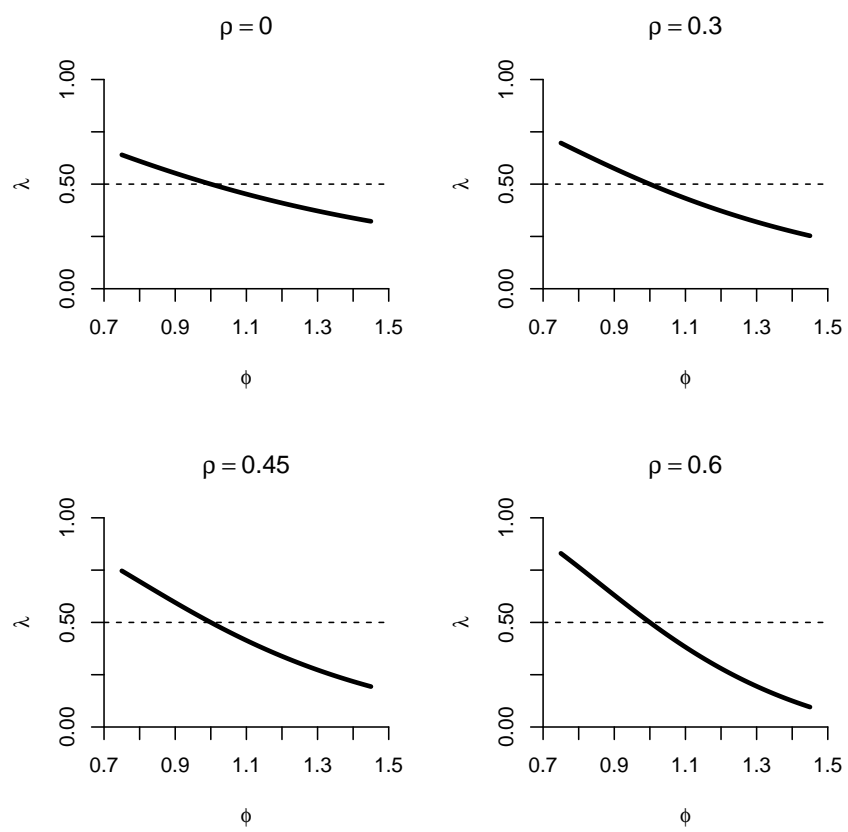

Figure 3: $\lambda^{*}$ vs. $\phi$ for Various $\rho$ Values. The horizontal line for visual reference is at $\lambda^{*}=.5$. See text for details.

Solving (5) for $\operatorname{cov}\left[G D P_{j}, G D P\right]$ and inserting the resulting expressions for $j \in\{E, I\}$ into (6) yields

$$
\sigma_{E I}=\operatorname{cov}\left[G D P_{I}, G D P_{E}\right]-\frac{1}{2}\left(\operatorname{var}\left[G D P_{I}\right]+\operatorname{var}\left[G D P_{E}\right]-\sigma_{I}^{2}-\sigma_{E}^{2}\right)
$$

Finally, let $\sigma_{E I}=\rho \sigma_{E} \sigma_{I}$ and $\sigma_{E}^{2}=\phi^{2} \sigma_{I}^{2}$. Then we can solve (7) for $\sigma_{I}^{2}$ :

$$
\sigma_{I}^{2}=\frac{\operatorname{cov}\left[G D P_{I}, G D P_{E}\right]-\frac{1}{2}\left(\operatorname{var}\left[G D P_{I}\right]+\operatorname{var}\left[G D P_{E}\right]\right)}{\rho \phi-\frac{1}{2}\left(1+\phi^{2}\right)}=\frac{N}{D} .
$$

For given values of $\phi$ and $\rho$ we can immediately evaluate the denominator $D$ in (8), and using data-based estimates of $\operatorname{cov}\left[G D P_{I}, G D P_{E}\right], \operatorname{var}\left[G D P_{I}\right]$ and $\operatorname{var}\left[G D P_{E}\right]$ we can evaluate the numerator $N$.

Based on our judgment regarding U.S. GDP $P_{E}$ and $G D P_{I}$ data (and again, we will discuss that judgment in detail in section 2.2), we believe that a reasonable range for $\rho$ is $\rho \in$ $[0.30,0.60]$, with midpoint 0.45 . One could think of this as a quasi-Bayesian statement that prior beliefs regarding $\rho$ are centered at 0.45 , with a ninety percent prior credible interval of 

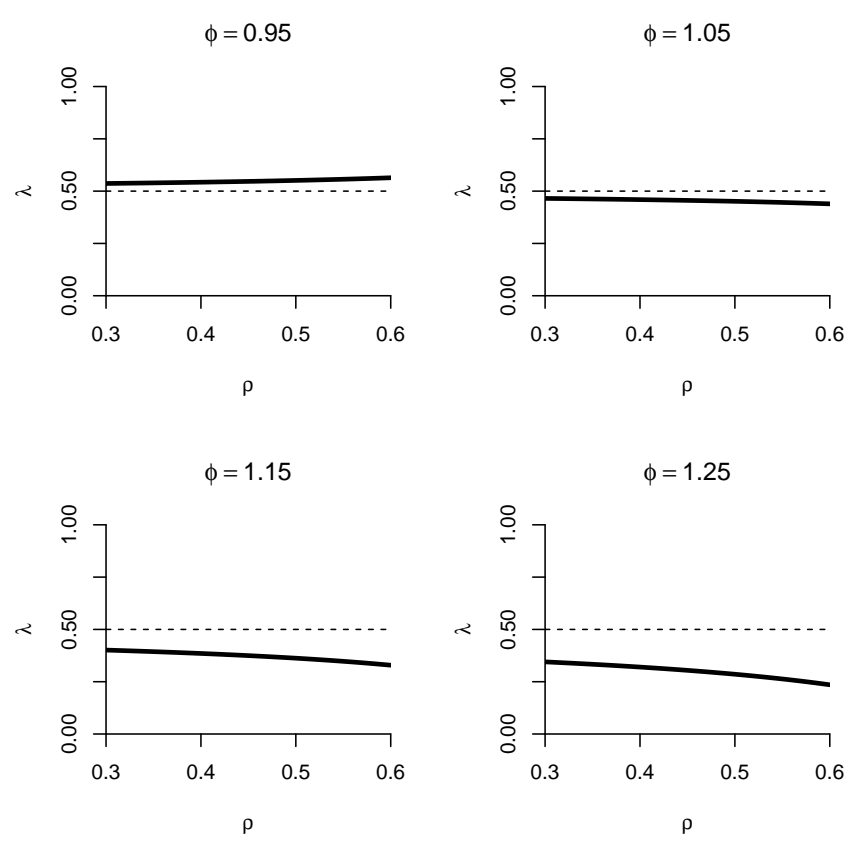

Figure 4: $\lambda^{*}$ vs. $\rho$ for Various $\phi$ Values. The horizontal line for visual reference is at $\lambda^{*}=.5$. See text for details.

$[0.30,0.60]^{6}$

In Figure 3 we show $\lambda^{*}$ as a function of $\phi$ for $\rho=0,0.3,0.45$ and 0.6 , in Figure 4 we show $\lambda^{*}$ as a function of $\rho$ for $\phi=0.95,1.05,1.15$ and 1.25, and in Figure 5 we show $\lambda^{*}$ as a bivariate function of $\phi$ and $\rho$. For $\phi=1$ the optimal weight is 0.5 for all $\rho$, but for $\phi \neq 1$ the optimal weight differs from 0.5 and is more sensitive to $\phi$ as $\rho$ grows. The crucial observation remains, however, that under a wide range of conditions it is optimal to put significant weight on both $G D P_{E}$ and $G D P_{I}$, with the optimal weights not differing radically from equality. Moreover, for all $\phi$ values greater than one, so that less weight is optimally placed on $G D P_{E}$ under a zero-correlation assumption, allowance for positive correlation further decreases the optimal weight placed on $G D P_{E}$. For a benchmark calibration of $\phi=1.1$ and $\rho=0.45$, $\lambda^{*} \approx 0.41$.

Let us again compare $\sigma_{C}^{2}$ to $\sigma_{E}^{2}$ for a range of $\lambda$ values (including $\lambda=\lambda^{*}, \lambda=0$, and

${ }^{6}$ Again using $G D P_{E}$ and $G D P_{I}$ data 1947Q2-2009Q3, we obtain for the numerator $N=-1.87$ in equation (7) above. And using the benchmark values of $\phi=1.1$ and $\rho=0.45$, we obtain for the denominator $D=-0.61$. This implies $\sigma_{I}=1.75$ and $\sigma_{E}=1.92$. For comparison, the standard deviation of $G D P_{E}$ and $G D P_{I}$ growth rates is about 4.2. Hence our benchmark calibration implies that the error in measuring true GDP by the reported $G D P_{E}$ and $G D P_{I}$ growth rates is potentially quite large. 


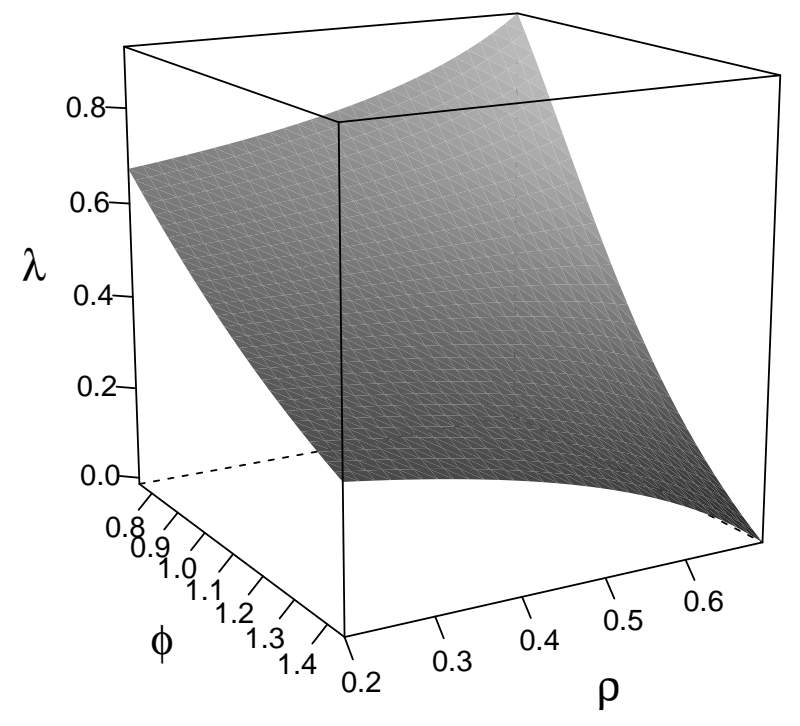

Figure 5: $\lambda^{*}$ vs. $\rho$ and $\phi$. See text for details.

$\lambda=1)$. From (4) we have:

$$
\frac{\sigma_{C}^{2}}{\sigma_{E}^{2}}=\lambda^{2}+\frac{(1-\lambda)^{2}}{\phi^{2}}+2 \lambda(1-\lambda) \frac{\rho}{\phi}
$$

In Figure 6 we graph $\sigma_{C}^{2} / \sigma_{E}^{2}$ for $\lambda \in[0,1]$ with $\phi=1.1$ and $\rho=0.45$. Obviously the maximum variance reduction is obtained using $\lambda^{*}=0.41$, but even for non-optimal $\lambda$, such as simple equal-weight combination $(\lambda=0.5)$, we achieve substantial variance reduction relative to using $G D P_{E}$ alone.

\subsection{On the Rationale for our Calibration}

We have thus far implicitly asked the reader to defer to our judgment regarding calibration, focusing on $\phi \in[.75,1.45]$ and $\rho \in[0.30,0.60]$ with benchmark midpoint values of $\phi=1.10$ and $\rho=0.45$. Here we explain the experience, reasoning, and research that supports that judgment. 


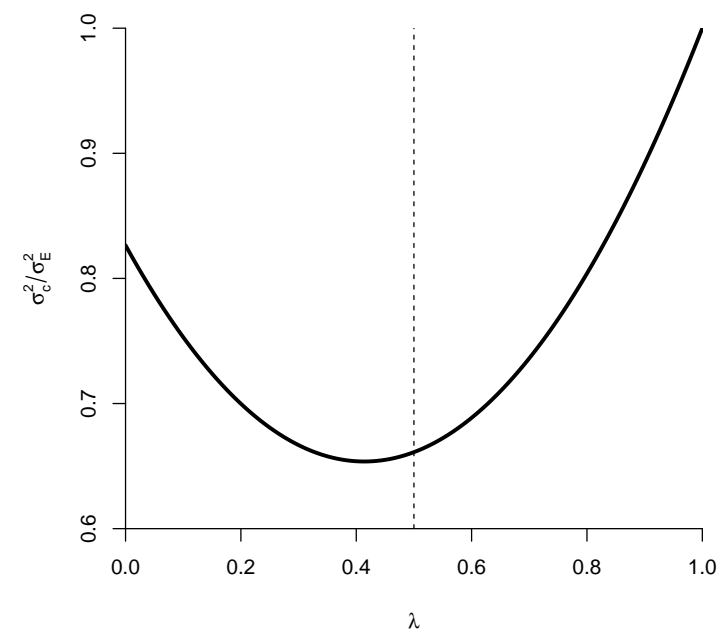

Figure 6: $\sigma_{C}^{2} / \sigma_{E}^{2}$ for $\lambda \in[0,1]$. We assume $\phi=1.1$ and $\rho=0.45$. See text for details.

\subsubsection{Calibrating $\phi$}

The key prior view embedded in our choice of $\phi \in[.75,1.45]$, with midpoint 1.10 , is that $G D P_{I}$ is likely a somewhat more accurate estimate than $G D P_{E}$. This accords with the results of Nalewaik (2010), who examines the relative accuracy of the $G D P_{E}$ and $G D P_{I}$ in several ways, with results favorable to $G D P_{I}$, suggesting $\phi>1$.

Let us elaborate. The first source of information on likely values of $\phi$ is from detailed examination of the source data underlying $G D P_{E}$ and $G D P_{I}$. The largest component of $G D P_{I}$, wage and salary income, is computed using quarterly data from tax records that are essentially universe counts, contaminated by neither sampling nor non-sampling errors. Two other very important components of $G D P_{I}$, corporate profits and proprietors' income, are also computed using annual data from tax records. ${ }^{7}$ Underreporting and non-reporting of income on tax forms (especially by proprietors) is an issue with these data, but the statistical agencies make adjustments for misreporting, and in any event the same misreporting issues plague $G D P_{E}$ as well as $G D P_{I}$, as we discuss below.

\footnotetext{
${ }^{7}$ The tax authorities do not release the universe counts for corporate profits and proprietors' income; rather, they release results from a random sample of tax returns. But the sample they employ is enormous, so the variance of the sampling error is tiny for the top-line estimates. Moreover, the tax authorities obviously know the universe count, so it seems unlikely that they would release tabulations that are very different from the universe counts.
} 
In contrast to $G D P_{I}$, very little of the quarterly or annual data used to compute $G D P_{E}$ is based on universe counts. ${ }^{8}$ Rather, most of the quarterly $G D P_{E}$ source data is from business surveys where response is voluntary. Non-response rates can be high, potentially introducing important sample-selection effects that may, moreover, vary with the state of the business cycle. Much annual $G D P_{E}$ source data is from business surveys with mandatory response, but some businesses still do not respond to the surveys, and surely the auditing of these non-respondents is less rigorous than the auditing of tax non-filers. In addition, even the annual surveys do not attempt to collect data on some types of small businesses, particularly non-employer businesses (i.e. businesses with no employees). The statistical agencies attempt to correct some of these omissions by incorporating data from tax records (making underreporting and non-reporting of income on tax forms an issue for $G D P_{E}$ as well as $\left.G D P_{I}\right)$, but it is not entirely clear whether they adequately plug all the holes in the survey data.

Although these problems plague most categories of $G D P_{E}$, some categories appear moreseverely plagued. In particular, over most of history, government statistical agencies have collected annual source data on less than half of personal consumption expenditures (PCE) for services, a very large category comprising between a quarter and a half of the nominal value of $G D P_{E}$ over our sample. At the quarterly frequency, statistical agencies have collected even less source data on services PCE. ${ }^{9}$ For this reason, statistical agencies have been forced to cobble together less-reliable data from numerous non-governmental sources to estimate services PCE.

A second source of information on the relative reliability of $G D P_{E}$ and $G D P_{I}$ is the correlation of the two measures with other variables that should be correlated with output growth, as examined in Nalewaik (2010). Nalewaik (2010) is careful to pick variables that are not used in the construction of either $G D P_{E}$ or $G D P_{I}$, to avoid spurious correlation resulting from correlated measurement errors. ${ }^{10}$ The results are uniformly favorable to $G D P_{I}$ and suggest that it is a more accurate measure of output growth than $G D P_{E}$. In particular, from the mid-1980s to the mid-2000s, the period of maximum divergence between $G D P_{E}$ and $G D P_{I}$, Nalewaik (2010) finds that $G D P_{I}$ growth has higher correlation with lagged stock price changes, the lagged slope of the yield curve, the lagged spread between high-

\footnotetext{
${ }^{8}$ Motor vehicle sales are a notable exception.

${ }^{9}$ This has begun to change recently, as the Census Bureau has expanded its surveys, but $\phi$ is meant to represent the average relative reliability over the sample we employ, so these facts are highly relevant.

${ }^{10}$ For example, the survey of households used to compute the unemployment rate is used in the construction of neither $G D P_{E}$ nor $G D P_{I}$, so use of variables from that survey is fine.
} 
yield corporate bonds and treasury bonds, short and long differences of the unemployment rate (both contemporaneously and at leads and lags), a measure of employment growth computed from the same household survey, the manufacturing ISM PMI (Institute for Supply Management, Purchasing Managers Index) the non-manufacturing ISM PMI, and dummies for NBER recessions. In addition, lags of $G D P_{I}$ growth also predict $G D P_{E}$ growth (and $G D P_{I}$ growth) better than lags of $G D P_{E}$ growth itself.

It is worth noting that, as regards our benchmark midpoint calibration of $\phi=1.10$, we have deviated only slightly from a "ignorance prior" midpoint of 1.00 . Hence our choice of midpoint reflects a conservative interpretation of the evidence discussed above. Similarly, regarding the width of the credible interval as opposed to its midpoint, we considered employing intervals such as $\phi \in[.95,1.25]$, for which $\phi>1$ over most of the mass of the interval. The evidence discussed above, if interpreted aggressively, might justify such a tight interval in favor of $G D P_{I}$, but again we opted for a more conservative approach with $\phi<1$ over more than a third of the mass of the interval.

\subsubsection{Calibrating $\rho$}

The key prior view embedded in our choice of $\rho \in[0.30,0.60]$, with midpoint 0.45 , is that the errors in $G D P_{E}$ and $G D P_{I}$ are likely positively correlated, with a moderately but not extremely large correlation value. This again accords with the results in Nalewaik (2010), who shows that 26 percent of the nominal value of $G D P_{E}$ and $G D P_{I}$ is identical. Any measurement errors in that 26 percent will be perfectly correlated across the two estimates. Furthermore, $G D P_{E}$ and $G D P_{I}$ are both likely to miss fluctuations in output occurring in the underground or "gray" economy, transactions that do not appear on tax forms or government surveys. In addition, the same price deflator is used to convert $G D P_{E}$ and $G D P_{I}$ from nominal to real values, so any measurement errors in that price deflator will be perfectly correlated across the two estimates.

These considerations suggest the lower bound for $\rho$ should be well above zero, as reflected in our chosen interval. However, the evidence favoring an upper bound well below one is also quite strong, as also reflected in our chosen interval. First, and most obviously, the standard deviation of the difference between $G D P_{E}$ and $G D P_{I}$ is 1.9 percent, far from the 0.0 percent that would be the case if $\rho=1.0$. Second, as discussed in the previous subsection, the source data used to construct $G D P_{E}$ is quite different from the source data used to construct $G D P_{I}$, implying the measurement errors are likely to be far from perfectly correlated. 
Of course, $\rho$ could still be quite high if $G D P_{E}$ and $G D P_{I}$ were contaminated with enormous common measurement errors, as well as smaller, uncorrelated measurement errors. But if that were the case, $G D P_{E}$ and $G D P_{I}$ would fail to be correlated with other cyclicallysensitive variables such as the unemployment rate, as they both are. The $R^{2}$ values from regressions of the output growth measures on the change in the unemployment rate are each around 0.50 over our sample, suggesting that at least half of the variance of $G D P_{E}$ and $G D P_{I}$ is true variation in output growth, rather than measurement error. The standard deviation of the residual from these regressions is 2.81 percent using $G D P_{I}$ and 2.95 percent using $G D P_{E}$. For comparison, taking our benchmark value $\phi=1.1$ and our upper bound $\rho=0.6$ produces $\sigma_{I}=2.05$ and $\sigma_{E}=2.25$. Increasing $\rho$ to 0.7 produces $\sigma_{I}=2.36$ and $\sigma_{E}=2.60$, approaching the residual standard error from our regression. This seems like an unreasonably high amount of measurement error, since the explained variation from such a simple regression is probably not measurement error, and indeed some of the unexplained variation from the regression is probably also not measurement error. Hence the upper bound of 0.6 for $\rho$ seems about right.

\section{Combination Under Minimax Loss}

Here we take a more conservative perspective on forecast combination, solving a different but potentially important optimization problem. In particular, by solving a game between a benevolent scholar (the Econometrician) and a malevolent opponent (Nature), we obtain "minimax" combining weights, which produce the smallest chance of the worst outcome for the Econometrician.

Minimax combining weights are of interest for at least two reasons. First, minimax calculations are the central decision-theoretic approach for imposing conservatism, and minimax combining weights are therefore of intrinsic interest. Moreover, to the best of our knowledge, minimax forecast combination has not yet been considered in the literature.

Second, and of particular importance in the present context of $G D P_{E}$ and $G D P_{I}$ combination, it transpires that optimal minimax combining weights do not depend on properties of the forecast errors. In particular, knowledge or calibration of objects like $\phi$ and $\rho$ is unnecessary, enabling us to dispense with judgment, for better or worse. Instead, as we shall show, the minimax optimization determines the minimax combining weights completely. 
We obtain the minimax weights by solving for the Nash equilibrium in a two-player zerosum game. Nature chooses the properties of the forecast errors and the Econometrician chooses the combining weights $\lambda$. For expositional purposes, we begin with the case of uncorrelated errors, constraining Nature to choose $\rho=0$. To impose some constraints on the magnitude of forecast errors that Nature can choose, it is useful to re-parameterize the vector $\left(\sigma_{I}, \sigma_{E}\right)^{\prime}$ in terms of polar coordinates; that is, we let $\sigma_{I}=\psi \cos \varphi$ and $\sigma_{E}=\psi \sin \varphi$. We restrict $\psi$ to the interval $[0, \bar{\psi}]$ and let $\varphi \in[0, \pi / 2]$. Because $\cos ^{2} \varphi+\sin ^{2} \varphi=1$, the sum of the forecast error variances associated with $G D P_{E}$ and $G D P_{I}$ is constrained to be less than or equal to $\bar{\psi}^{2}$. The error associated with the combined forecast is given by

$$
\sigma_{C}^{2}(\psi, \varphi, \lambda)=\psi^{2}\left[\lambda^{2} \sin ^{2} \varphi+(1-\lambda)^{2} \cos ^{2} \varphi\right]
$$

so that the minimax problem is

$$
\max _{\psi \in[0, \bar{\psi}], \varphi \in[0, \pi / 2]} \min _{\lambda \in[0,1]} \sigma_{C}^{2}(\psi, \varphi, \lambda) .
$$

The best response of the Econometrician was derived in (2) and can be expressed in terms of polar coordinates as $\lambda^{*}=\cos ^{2} \varphi$. In turn, Nature's' problem simplifies to

$$
\max _{\psi \in[0, \bar{\psi}], \varphi \in[0, \pi / 2]} \psi^{2}\left(1-\sin ^{2} \varphi\right) \sin ^{2} \varphi
$$

which leads to the solution

$$
\varphi^{*}=\arcsin \sqrt{1 / 2}, \quad \psi^{*}=\bar{\psi}, \quad \lambda^{*}=1 / 2
$$

Nature's optimal choice implies a unit forecast error variance ratio, $\phi=\sigma_{E} / \sigma_{I}=1$, and hence that the optimal combining weight is $1 / 2$. If, instead, Nature set $\varphi=0$ or $\varphi=\pi / 2$, that is $\phi=0$ or $\phi=\infty$, then either $G D P_{E}$ or $G D P_{I}$ is perfect and the Econometrician could choose $\lambda=0$ or $\lambda=1$ to achieve a perfect forecast leading to a suboptimal outcome for Nature.

Now we consider the case in which Nature can choose a non-zero correlation between the forecast errors of $G D P_{E}$ and $G D P_{I}$. The loss of the combined forecast can be expressed as

$$
\sigma_{C}^{2}(\psi, \rho, \varphi, \lambda)=\psi^{2}\left[\lambda^{2} \sin ^{2} \varphi+(1-\lambda)^{2} \cos ^{2} \varphi+2 \lambda(1-\lambda) \rho \sin \varphi \cos \varphi\right]
$$


It is apparent from (12) that as long as $\lambda$ lies in the unit interval the most devious choice of $\rho$ is $\rho^{*}=1$. We will now verify that conditional on $\rho^{*}=1$ the solution in (11) remains a Nash Equilibrium. Suppose that the Econometrician chooses equal weights $\lambda^{*}=1 / 2$. In this case

$$
\sigma_{C}^{2}\left(\psi, \rho^{*}, \varphi, \lambda^{*}\right)=\psi^{2}\left[\frac{1}{4}+\frac{1}{2} \sin \varphi \cos \varphi\right] .
$$

We can deduce immediately that $\psi^{*}=\bar{\psi}$. Moreover, first-order conditions for the maximization with respect to $\varphi$ imply that $\cos ^{2} \varphi^{*}=\sin ^{2} \varphi^{*}$ which in turn leads to $\varphi^{*}=\operatorname{arc} \sin \sqrt{1 / 2}$. Conditional on Nature choosing $\rho^{*}, \psi^{*}$, and $\varphi^{*}$, the Econometrician has no incentive to deviate from the equal-weights combination $\lambda^{*}=1 / 2$, because

$$
\sigma_{C}^{2}\left(\psi^{*}, \rho^{*}, \varphi^{*}, \lambda\right)=\frac{\bar{\psi}}{2}\left[\lambda^{2}+(1-\lambda)^{2}+2 \lambda(1-\lambda)\right]=\frac{\bar{\psi}}{2}
$$

In sum, the minimax analysis provides a rational for combining $G D P_{E}$ and $G D P_{I}$ with equal weights of $\lambda=1 / 2$.

\section{Empirics}

We have shown that combining using a quasi-Bayesian calibration under quadratic loss produces $\lambda$ close to but less than 0.5 , given our prior means for $\phi$ and $\rho$. Moreover, we showed that combining with $\lambda$ near 0.5 is likely better - often much better - than simply using $G D P_{E}$ or $G D P_{I}$ alone, for wide ranges of $\phi$ and $\rho$. We also showed that combining under minimax loss always implies an optimal $\lambda$ of exactly 0.5 .

Here we put the theory to work for the U.S., providing arguably-superior combined estimates of GDP growth. We focus on quasi-Bayesian calibration under quadratic loss. Because the resulting combining weights are near 0.50 , however, one could also view our combinations as approximately minimax. The point is that a variety of perspectives lead to combinations with weights near 0.50 , and they suggest that such combinations are likely superior to using either of $G D P_{E}$ or $G D P_{I}$ alone, so that empirical examination of $G D P_{C}$ is of maximal interest. 

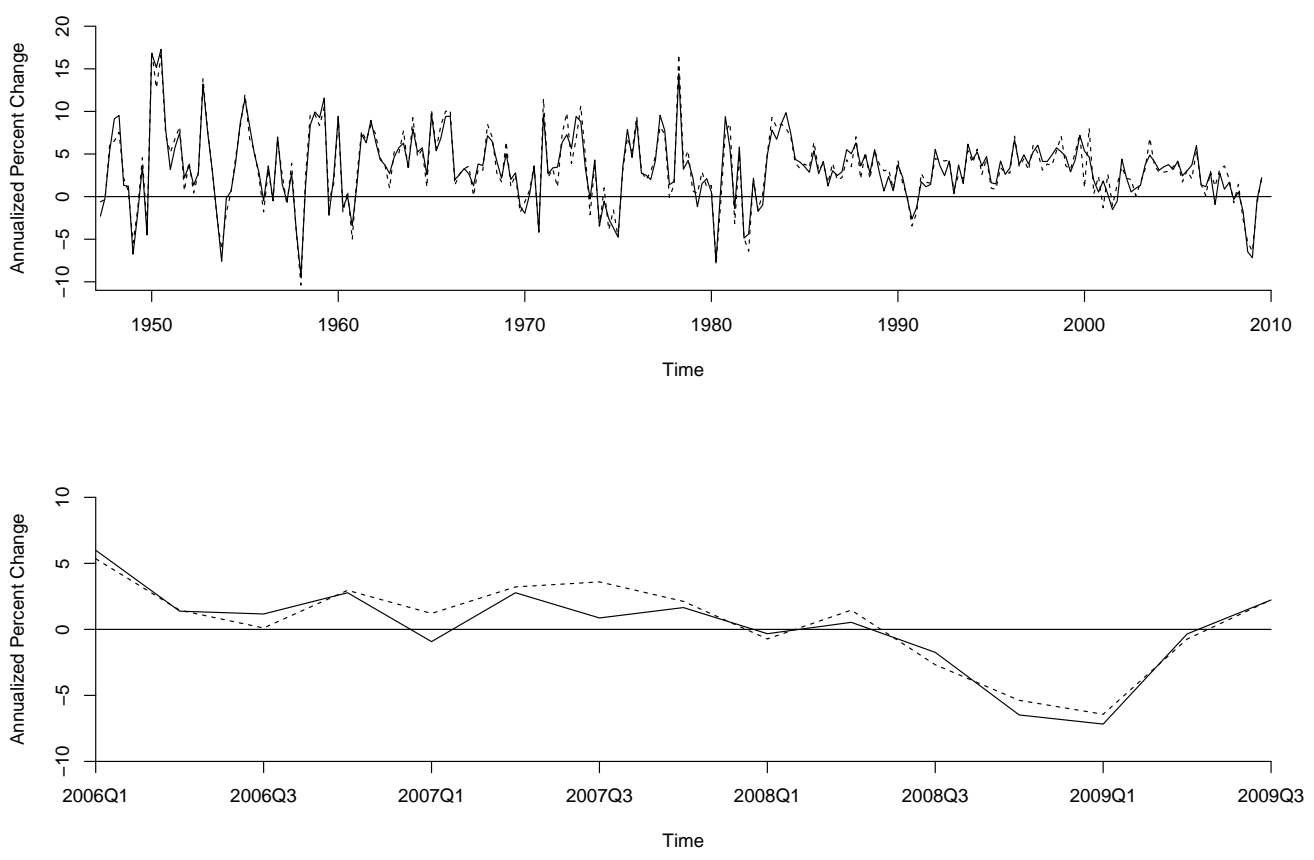

Figure 7: U.S. $G D P_{C}$ and $G D P_{E}$ Growth Rates. $G D P_{C}$ constructed assuming $\phi=1.1$ and $\rho=0.45$. $G D P_{C}$ is solid and $G D P_{E}$ is dashed. In the top panel we show a long sample, 1947Q2-2009Q3. In the bottom panel, we show a recent sample, 2006Q1-2009Q3. See text for details.

\subsection{A Combined U.S. GDP Series}

In the top panel of Figure 7 we plot $G D P_{C}$ constructed using $\lambda=0.41$, which is optimal for our benchmark calibration of $\phi=1.1$ and $\rho=0.45$, together with the "conventional" $G D P_{E}$. The two appear to move closely together, and indeed they do, at least at the low frequencies emphasized by the long time-series plot. Hence for low-frequency analyses, such as studies of long-term economic growth, use of $G D P_{E}, G D P_{I}$ or $G D P_{C}$ is not likely to make a major difference.

At higher frequencies, however, important divergences can occur. In the bottom panel of Figure 7, for example, we emphasize business cycle frequencies by focusing on a short sample 2006-2010, which contains the severe U.S. recession of 2007-2009. There are two important points to notice. First, the bottom panel of Figure 7 makes clear that growth-rate assessments on particular dates can differ in important ways depending on whether $G D P_{C}$ or $G D P_{E}$ is used. For example, $G D P_{E}$ is strongly positive for 2007Q3, whereas $G D P_{C}$ for that quarter is close to zero, as $G D P_{I}$ was strongly negative. Second, the bottom panel 

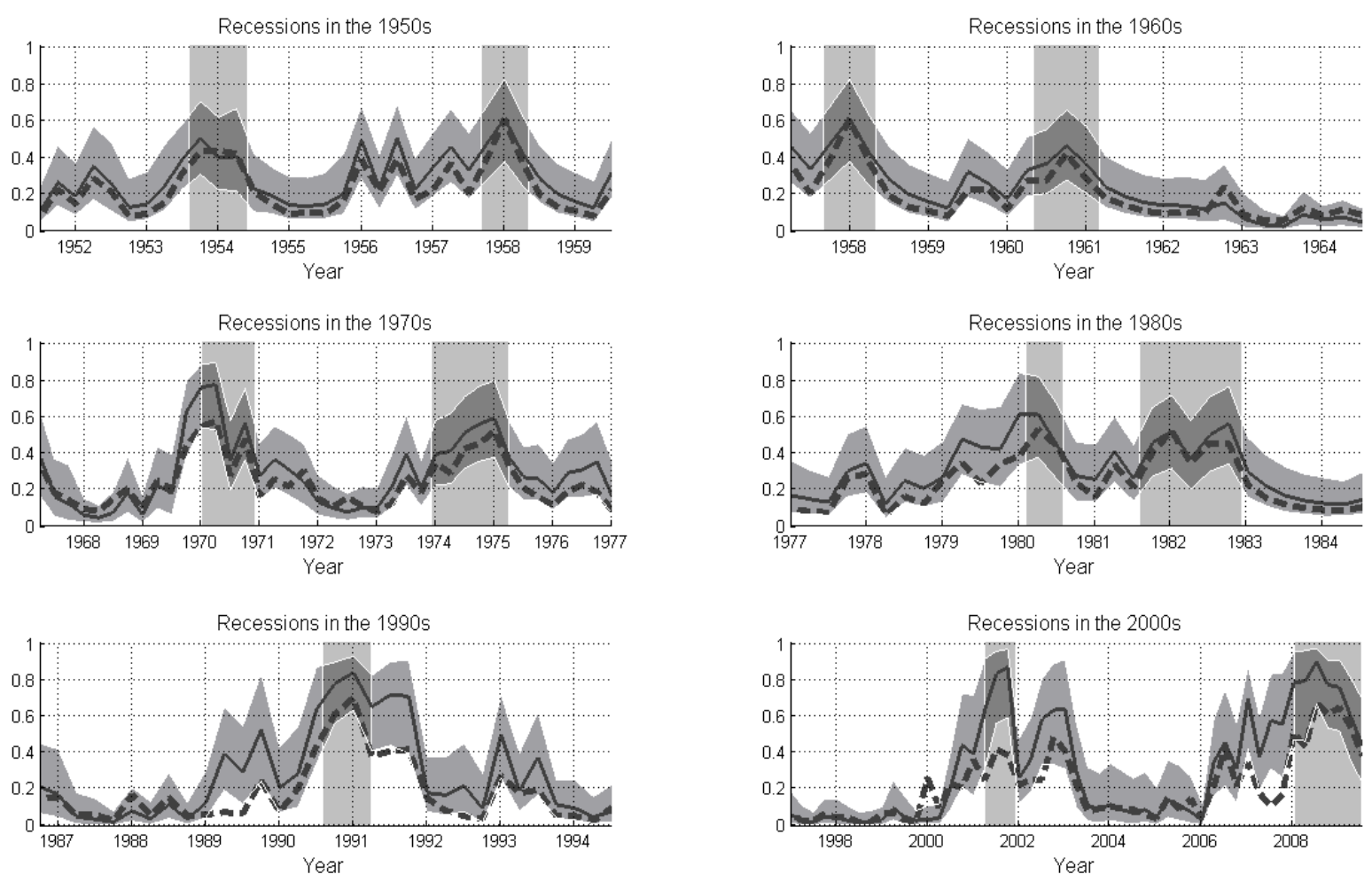

Figure 8: Inferred U.S. Recession Regime Probabilities, Calculated Using $G D P_{C}$ vs. $G D P_{E}$. Solid lines are posterior median smoothed recession regime probabilities calculated using $G D P_{C}$, which we show with ninety percent posterior intervals. Dashed lines are posterior median smoothed recession regime probabilities calculated using $G D P_{E}$. Sample period is 1947Q2-2009Q3. Dark shaded bars denote NBER recessions. See text and appendix for details.

of Figure 7 also makes clear that differing assessments can persist over several quarters, as for example during the financial crisis episode of 2007Q1-2007Q3, when $G D P_{E}$ growth was consistently larger than $G D P_{C}$ growth. One might naturally conjecture that such persistent and cumulative data distortions might similarly distort inferences, based on those data, about whether and when the U.S. economy was in recession. We now consider recession dating in some detail.

\subsection{U.S. Recession and Volatility Regime Probabilities}

Thus far we have assessed how combining produces changes in measured GDP. Now we assess whether and how it changes a certain important transformation of GDP, namely measured probabilities of recession regimes or high-volatility regimes based on measured GDP. We 
proceed by fitting a regime-switching model in the tradition of Hamilton (1989), generalized to allow for switching in both means and variances, as in Kim and Nelson (1999a),

$$
\begin{gathered}
\left(G D P_{t}-\mu_{s_{\mu t}}\right)=\beta\left(G D P_{t-1}-\mu_{s_{\mu t-1}}\right)+\sigma_{s_{\sigma t}} \varepsilon_{t} \\
\varepsilon_{t} \sim \operatorname{iidN}(0,1) \\
s_{\mu t} \sim \operatorname{Markov}\left(P_{\mu}\right), \quad s_{\sigma t} \sim \operatorname{Markov}\left(P_{\sigma}\right) .
\end{gathered}
$$

Then, conditional on observed data, we infer the sequences of recession probabilities $\left(P\left(s_{\mu t}=\right.\right.$ $L)$, where $L$ ("low") denotes the recession regime) and high-volatility regime probabilities $\left(P\left(s_{\sigma_{t}}=H\right)\right.$, where $H$ ("high") denotes the high-volatility regime). We perform this exercise using both $G D P_{E}$ and $G D P_{C}$, and we compare the results.

We implement Bayesian estimation and state extraction using data 1947Q2-2009Q3. ${ }^{11}$ In Figure 8 we show posterior median smoothed recession probabilities. We show those calculated using $G D P_{C}$ as solid lines with ninety percent posterior intervals, we show those calculated using $G D P_{E}$ as dashed lines, and we also show shaded NBER recession episodes to help provide context. Similarly, in Figure 9 we show posterior median smoothed volatility regime probabilities.

Numerous interesting substantive results emerge. For example, posterior median smoothed recession regime probabilities calculated using $G D P_{C}$ tend to be greater than those calculated using $G D P_{E}$, sometimes significantly so, as for example during the financial crisis of 2007. Indeed using $G D P_{C}$ one might date the start of the recent recession significantly earlier than did the NBER. As regards volatilities, posterior median smoothed high-volatility regime probabilities calculated by either $G D P_{E}$ or $G D P_{C}$ tend to show the post-1984 "great moderation" effect asserted by McConnell and Perez-Quiros (2000) and Stock and Watson (2002). Interestingly, however, those calculated using $G D P_{E}$ also show the "higher recession volatility" effect in recent decades documented by Bloom et al. (2009) (using GDP $P_{E}$ data), whereas those calculated using $G D P_{C}$ do not.

For our present purposes, however, none of those substantive results are of first-order importance, as the present paper is not about business cycle dating, low-frequency vs. highfrequency volatility regime dating, or revisionist history, per se. Indeed thorough explorations of each would require separate and lengthy papers for each. Rather, our point here is simply

\footnotetext{
${ }^{11}$ We provide a detailed description in Appendix A.
} 

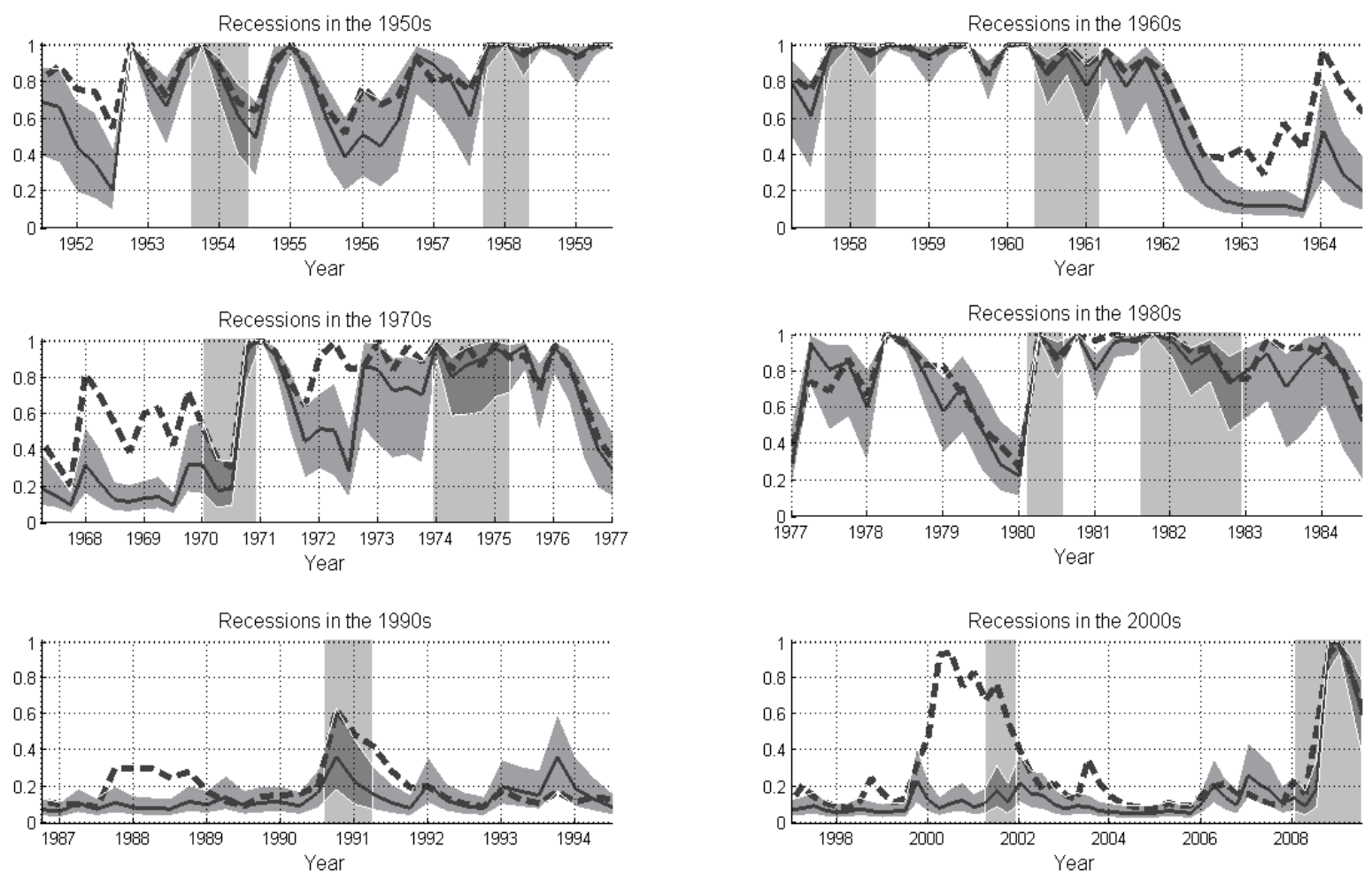

Figure 9: Inferred U.S. High-Volatility Regime Probabilities, Calculated Using $G D P_{C}$ vs. $G D P_{E}$. Solid lines are posterior median smoothed high-volatility regime probabilities calculated using $G D P_{C}$, which we show with ninety percent posterior intervals. Dashed lines are posterior median smoothed high-volatility regime probabilities calculated using $G D P_{E}$. Sample period is 1947Q2-2009Q3. Dark shaded bars denote NBER recessions. See text and appendices for details.

that one's assessment and characterization of macroeconomic behavior can, and often does, depend significantly on use of $G D P_{C}$ vs. $G D P_{E}$. That is, choice of $G D P_{C}$ vs. $G D P_{E}$ can matter for important tasks, whether based on direct observation of measured GDP, or on transformations of measured GDP such as extracted regime chronologies.

\section{Extensions}

Before concluding, we offer sketches of what we see as two important avenues for future research. The first involves real-time analysis and non-constant combining weights, and the second involves combining from a measurement error as opposed to efficient forecast error perspective. 


\subsection{Vintage Data, Time-Varying Combining Weights, and Real- Time Analysis}

It is important to note that everything that we have done in this paper has a retrospective, or "off-line," character. We work with a single vintage of $G D P_{E}$ and $G D P_{I}$ data and combine them, estimating objects of interest (combining weights, regime probabilities, etc.) for any period $t$ using all data $t=1, \ldots, T$. In all of our analysis, moreover, we have used time-invariant combining weights. Those two characteristics of our work thus far are not unrelated, and one may want to relax them eventually, allowing for time-varying weights, and ultimately, a truly real-time-analysis.

One may want to consider time-varying combining weights for several reasons. One reason is of near-universal and hence great interest, at least under quadratic loss. For any given vintage of data, error variances and covariances may naturally change, as we pass backward from preliminary data for the recent past, all the way through to "final revised" data for the more distant past. ${ }^{12}$ More precisely, let $t$ index time measured in quarters, and consider moving backward from "the present" quarter $t=T$. At instant $v \in T$ (with apologies for the slightly abusive notation), we have vintage- $v$ data. Consider moving backward, constructing combined GDP estimates $G D P_{C, T-k}^{v}, k=1, \ldots \infty$. For small $k$, the optimal calibrations might be quite far from benchmark values. As $k$ grows, however, $\rho$ and $\phi$ should approach benchmark values as the final revision is approached. The obvious question is how quickly and with what pattern should an optimal calibration move toward benchmark values as $k \rightarrow \infty$. We can offer a few speculative observations.

First consider $\rho$. GDP $P_{I}$ and $G D P_{E}$ share a considerable amount of source data in their early releases, before common source data is swapped out of $G D P_{I}$ (e.g., when tax returns eventually become available and can be used). Indeed Fixler and Nalewaik (2009) show that the correlation between the earlier estimates of $G D P_{I}$ and $G D P_{E}$ growth is higher than the correlation between the later estimates. Hence $\rho$ is likely higher for dates near the present (small $k$ ). This suggests calibrations with $\rho$ dropping monotonically toward the benchmark value of 0.45 as $k$ grows.

Now consider $\phi$. How $\phi$ should deviate from its benchmark calibration value of 1.1 is less clear. On the one hand, early releases of $G D P_{I}$ are missing some of its most informative source data (tax returns), which suggests a lower-than-benchmark $\phi$ for small $k$. On the

\footnotetext{
${ }^{12}$ This is the so-called "apples and oranges" problem. To the best of our knowledge, the usage in our context traces to Kishor and Koenig (2011).
} 
other hand, early releases of $G D P_{E}$ growth appear to be noisier than the early releases of $G D P_{I}$ growth (see below), which suggests a higher-than-benchmark $\phi$ for small $k$. All told, we feel that a reasonable small- $k$ calibration of $\phi$ is less than 1.1 but still above 1 .

Note that our conjectured small- $k$ effects work in different directions. Other things equal, bigger $\rho$ pushes the optimal combining weight downward, away from 0.5 , and smaller $\phi$ pushes the optimal combining weight upward, toward from 0.5. In any particular dataset the effects could conceivably offset more-or-less exactly, so that combination using constant weights for all dates would be fully optimal, but there is of course no guarantee.

Several approaches are possible to implement the time-varying weights sketched in the preceding paragraphs. One is a quasi-Bayesian calibration, elaborating on the approach we have taken in this paper. However, such an approach would be more difficult in the more challenging environment of time-varying parameters. Another is to construct a real-time dataset, one that records a snapshot of the data available at each point in time, such as the one maintained by the Federal Reserve Bank of Philadelphia. The key is to recognize that each quarter we get not simply one new observation on $G D P_{E}$ and $G D P_{I}$, but rather an entire new vintage of data, all the elements of which could (in principle) change. One might be able to use the different data vintages, and related objects like revision histories, to infer properties of "forecast errors" of relevance for construction of optimal combining weights across various $k$.

One could go even farther in principle, progressing to a truly real-time analysis, which is of intrinsic interest quite apart from addressing the issue of time-varying combining weights in the above "apples and oranges" environments. Tracking vintages, modeling the associated dynamics of revisions, and putting it all together to produce superior combined forecasts remains an outstanding challenge. ${ }^{13}$ We look forward to its solution in future work, potentially in the state-space framework that we describe next.

\subsection{A Model of Measurement Error}

In parallel work in progress, Aruoba et al. (2011), we pursue a complementary approach based on a state-space model of measurement error. The basic model is

\footnotetext{
${ }^{13}$ Nalewaik (2011) makes some progress toward real-time analysis in a Markov-switching environment.
} 


$$
\begin{gathered}
\left(\begin{array}{c}
G D P_{E, t} \\
G D P_{I, t}
\end{array}\right)=\left(\begin{array}{l}
1 \\
1
\end{array}\right) \mathrm{GDP}_{\mathrm{t}}+\left(\begin{array}{l}
\varepsilon_{E t} \\
\varepsilon_{I t}
\end{array}\right) \\
\mathrm{GDP}_{\mathrm{t}}=\beta_{0}+\beta_{1} \mathrm{GDP}_{\mathrm{t}-1}+\eta_{\mathrm{t}},
\end{gathered}
$$

where $\varepsilon_{t}=\left(\varepsilon_{E t}, \varepsilon_{I t}\right)^{\prime} \sim W N\left(\underline{0}, \Sigma_{\varepsilon}\right), \eta_{t} \sim W N\left(0, \sigma_{\eta}^{2}\right)$, and $\varepsilon_{t}$ and $\eta_{t}$ are uncorrelated at all leads and lags. In this model, both $G D P_{E}$ and $G D P_{I}$ are noisy measures of the latent true GDP process, which evolves dynamically. The expectation of true GDP conditional upon observed measurements may be extracted using optimal filtering techniques such as the Kalman filter.

The basic state-space model can be extended in various directions, for example to incorporate richer dynamics, and to account for data revisions and missing advance and preliminary releases of $G D P_{I} \cdot{ }^{14}$ Perhaps most importantly, the measurement errors $\varepsilon$ may be allowed to be correlated with GDP, or more precisely, correlated with GDP innovations, $\eta_{t}$. Fixler and Nalewaik (2009) and Nalewaik (2010) document cyclicality in the "statistical discrepancy" $\left(G D P_{E}-G D P_{I}\right)$, which implies failure of the assumption that $\varepsilon_{t}$ and $\eta_{t}$ are uncorrelated at all leads and lags. Of particular concern is contemporaneous correlation between $\eta_{t}$ and $\varepsilon_{t}$. The standard Kalman filter can not handle this, but appropriate modifications are available.

\section{Conclusions}

GDP growth is a central concept in macroeconomics and business cycle monitoring, so its accurate measurement is crucial. Unfortunately, however, the two available expenditure-side and income-side U.S. GDP estimates often diverge. In this paper we proposed a technology for optimally combining the competing GDP estimates, we examined several variations on the basic theme, and we constructed and examined combined estimates for the U.S.

Our results strongly suggest the desirability of separate and careful calculation of both $G D P_{E}$ and $G D P_{I}$, followed by combination, which may lead to different and more accurate insights than those obtained by simply using expenditure-side or estimates alone. This

\footnotetext{
${ }^{14}$ The first official estimate of $G D P_{I}$ is released a month or two after the first official estimate of $G D P_{E}$, so for vintage $v$ the available $G D P_{E}^{v}$ data might be $\left\{G D P_{E, t}^{v}\right\}_{t=1}^{T-1}$ whereas the available $G D P_{I}^{v}$ vintage might be $\left\{G D P_{I, t}^{v}\right\}_{t=1}^{T-2}$. Note that for any vintage $v$, the available $G D P_{I}$ data differs by at most one quarter from the available $G D P_{E}$ data.
} 
prescription differs fundamentally from U.S. practice, where both are calculated but the income-side estimate is routinely ignored.

Our call for a combined U.S. GDP measure is hardly radical, particularly given current best-practice procedures at various non-U.S. statistical agencies. European countries, for example, tend to use sophisticated GDP balancing procedures to harmonize GDP estimates from different sources. ${ }^{15}$ The balancing procedure recognizes the potential inaccuracies of source data and has a similar effect to our forecast combination approach: the final GDP number lies between the alternative estimates. Other countries use other approaches to combination. Strikingly, for example, Australia uses an approach reminiscent of the one that we advocate in this paper, albeit not on the grounds of our formal analysis. ${ }^{16}$ In addition to $G D P_{E}$ and $G D P_{I}$, the Australian Bureau of Statistics produces a productionside estimate, $G D P_{P}$, defined as total gross value added plus taxes and less subsidies, and its headline GDP number is the simple average of the three GDP estimates. We look forward to the U.S. producing a similarly-combined headline GDP estimate, potentially using the methods introduced in this paper.

\footnotetext{
${ }^{15}$ Germany's procedures, for example, are described in Statistisches Bundesamt (2009).

${ }^{16}$ See http://www.abs.gov.au, under Australian National Accounts, Explanatory Notes for Australia.
} 


\section{Appendices}

\section{A Estimation of U.S. Recession Probabilities}

Here we provide details of Bayesian analysis of our regime-switching model.

\section{A.1 Baseline Model}

We work with a simple model with Markov regime-switching in mean and variance:

$$
\begin{gathered}
\left(G D P_{t}-\mu_{s_{\mu t}}\right)=\beta\left(G D P_{t-1}-\mu_{s_{\mu t-1}}\right)+\sigma_{s_{\sigma t}} \varepsilon_{t} \\
\varepsilon_{t} \sim \operatorname{iidN}(0,1) \\
s_{\mu t} \sim \operatorname{Markov}\left(P_{\mu}\right), \quad s_{\sigma t} \sim \operatorname{Markov}\left(P_{\sigma}\right),
\end{gathered}
$$

where $P_{\mu}$ and $P_{\sigma}$ denote transition matrices for high and low mean and variance regimes,

$$
\begin{gathered}
P_{\mu}=\left[\begin{array}{cc}
p_{\mu_{H}} & 1-p_{\mu_{H}} \\
1-p_{\mu_{L}} & p_{\mu_{L}}
\end{array}\right] \\
P_{\sigma}=\left[\begin{array}{cc}
p_{\sigma_{H}} & 1-p_{\sigma_{H}} \\
1-p_{\sigma_{L}} & p_{\sigma_{L}}
\end{array}\right] .
\end{gathered}
$$

Overall, then, there are four regimes:

$$
\begin{aligned}
& S_{t}=1 \quad \text { if } \quad s_{\mu t}=H, s_{\sigma t}=H \\
& S_{t}=2 \quad \text { if } \quad s_{\mu t}=H, s_{\sigma t}=L \\
& S_{t}=3 \quad \text { if } \quad s_{\mu t}=L, s_{\sigma t}=H \\
& S_{t}=4 \quad \text { if } \quad s_{\mu t}=L, s_{\sigma t}=L .
\end{aligned}
$$

For $t=0$ the hidden Markov states are governed by the ergodic distribution associated with $P_{\mu}$ and $P_{\sigma}$. 
Table 1: Prior Choices and Posterior Distributions

\begin{tabular}{cccccccc}
\hline & Prior & \multicolumn{3}{c}{$G D P_{E}$} & \multicolumn{3}{c}{$G D P_{C}$} \\
& Choice & Median & $5 \%$ & $95 \%$ & Median & $5 \%$ & $95 \%$ \\
\hline$\mu_{H}-\mu_{L}$ & Gamma(2,1) & - & - & - & - & - & - \\
$\mu_{H}$ & - & 3.50 & {$[3.03$} & $4.12]$ & 3.76 & {$[2.97$} & $4.28]$ \\
$\mu_{L}$ & Normal(0,0.5) & 1.25 & {$[0.34$} & $2.29]$ & 0.82 & {$[0.17$} & $1.64]$ \\
$\sigma_{H}$ & InvGamma(2,2) & 4.82 & {$[4.35$} & $5.43]$ & 4.64 & {$[4.21$} & $5.13]$ \\
$\sigma_{L}$ & InvGamma(1,2) & 1.92 & {$[1.55$} & $2.34]$ & 1.71 & {$[1.74$} & $2.05]$ \\
$\beta$ & Normal(0,1) & 0.31 & {$[0.17$} & $0.45]$ & 0.37 & {$[0.27$} & $0.53]$ \\
$p_{\mu_{H}}$ & $\operatorname{Beta}(25,5)$ & 0.91 & {$[0.82$} & $0.96]$ & 0.92 & {$[0.85$} & $0.96]$ \\
$p_{\mu_{L}}$ & $\operatorname{Beta}(25,5)$ & 0.79 & {$[0.64$} & $0.87]$ & 0.80 & {$[0.67$} & $0.88]$ \\
$p_{\sigma_{H}}$ & $\operatorname{Beta}(25,5)$ & 0.91 & {$[0.83$} & $0.96]$ & 0.91 & {$[0.83$} & $0.96]$ \\
$p_{\sigma_{L}}$ & $\operatorname{Beta}(25,5)$ & 0.89 & {$[0.81$} & $0.95]$ & 0.91 & {$[0.85$} & $0.95]$ \\
\hline
\end{tabular}

\section{A.2 Bayesian Inference}

Priors. Bayesian inference combines a prior distribution with a likelihood function to obtain a posterior distribution of the model parameters and states. We summarize our benchmark priors in Table 1 . We employ a normal prior for $\mu_{L}$, a gamma prior for $\mu_{H}-\mu_{L}$, inverted gamma priors for $\sigma_{H}$ and $\sigma_{L}$, beta priors for the transition probabilities, and finally, a normal prior for $\beta$. Our prior ensures that $\mu_{H} \geq \mu_{L}$ and thereby deals with the "label switching" identification problem.

For $\mu_{L}$, the average growth rate in the low-growth state, we use a prior distribution that is centered at 0 , with standard deviation $0.7 \%$. Note that a priori we do not restrict the average growth rate to be negative. We also allow for (mildly) positive values. Wh choose the prior for $\mu_{H}-\mu_{L}$ such that the mean difference between the average growth rates in the two regimes is $2 \%$, with standard deviation $1 \%$. Our priors for the transition probabilities $p_{\mu}$ and $p_{\sigma}$ are symmetric and imply a mean regime duration between 3 and 14 quarters. Finally, our choice for the prior of the autoregressive parameter $\beta$ is normal with zero mean and unit variance, allowing a priori for both stable and unstable dynamics of output growth rates.

Implementation of Posterior Inference. Posterior inference is implemented with a Metropolis-within-Gibbs sampler, building on work by Carter and Kohn (1994) and Kim and Nelson (1999b). We denote the sequence of observations by $G D P_{1: T}$. Moreover, let $S_{1: T}$ 
be the sequence of hidden states, and let

$$
\theta=\left(\mu_{H}, \mu_{L}, \sigma_{H}, \sigma_{L}, \beta\right)^{\prime}, \quad \text { and } \quad \phi=\left(p_{\mu_{H}}, p_{\mu_{L}}, p_{\sigma_{L}}, p_{\sigma_{H}}\right)^{\prime}
$$

Our Metropolis-within-Gibbs algorithm involves sampling iteratively from three conditional posterior distributions. To initialize the sampler we start from $\left(\theta^{0}, \phi^{0}\right)$.

\section{Algorithm: Metropolis-within-Gibbs Sampler}

For $i=1, \ldots, N$ :

1. Draw $S_{1: T}^{i+1}$ conditional on $\theta^{i}, \phi^{i}, G D P_{1: T}$. This step is implemented using the multimove simulation smoother described in Section 9.1.1 of Kim and Nelson (1999b).

2. Draw $\phi^{i+1}$ conditional on $\theta^{i}, S_{1: T}^{i+1}, G D P_{1: T}$. If the dependence of the distribution of the initial state $S_{1}$ on $\phi$ is ignored, then it can be shown that the conditional posterior of $\phi$ is of the Beta form (see Section 9.1.2 of Kim and Nelson (1999b)). We use the resulting Beta distribution as a proposal distribution in a Metropolis-Hastings step.

3. Draw $\theta^{i+1}$, conditional on $\phi^{i+1}, S_{1: T}^{i+1}, G D P_{1: T}$. Since our prior distribution is nonconjugate, we are using a random-walk Metropolis step to generate a draw from the conditional posterior of $\theta$. The proposal distribution is $N\left(\theta^{i}, c \Omega\right)$.

We obtain the covariance matrix $\Omega$ of the proposal distribution in Step 3 as follows. Following Schorfheide (2005) we maximize the posterior density,

$$
p\left(\theta, \phi \mid G D P_{1: T}\right) \propto p\left(G D P_{1: T} \mid \theta, \phi\right) p(\theta, \phi)
$$

to obtain the posterior mode $(\tilde{\theta}, \tilde{\phi})$. We then construct the negative inverse of the Hessian at the mode and let $\Omega$ be the sub-matrix that corresponds to the parameter sub-vector $\theta$. We choose the scaling factor $c$ to obtain an acceptance rate of approximately $40 \%$. We initialize our algorithm choosing $\left(\theta^{0}, \phi^{0}\right)$ in the neighborhood of $(\tilde{\theta}, \tilde{\phi})$ and use it to generate $N=100,000$ draws from the posterior distribution. ${ }^{17}$

Posterior Estimates. Table 1 also contains percentiles of posterior parameter distributions. The posterior estimates for the volatility parameters and the transition probabilities are similar across $G D P_{E}$ and $G D P_{C}$. However, the posterior estimate for $\mu_{L}$ is higher using

\footnotetext{
${ }^{17}$ We performed several tests confirming that our choice of $N$ yields an accurate posterior approximation.
} 
$G D P_{E}$ than using $G D P_{C}$, while the opposite is true for $\beta$. Moreover, the differential between high and low mean regimes is bigger in the case of $G D P_{C}$, all of which can influence the time-series plot of the recession probabilities.

The Markov-switching means capture low-frequency shifts while the autoregressive coefficient captures high-frequency dynamics. Thus, the presence of the autoregressive term may complicate our analysis, because we are trying to decompose the GDP measurement discrepancy into both low and high frequency components. As a robustness check, we remove the autoregressive term in (A.1) and estimate an iid model specification. Although the posterior estimates for $\mu_{L}$ change, the remaining parameters are essentially identical to Table 1 . The smoothed recession probabilities remain nearly identical to Figure 8. 


\section{References}

Aruoba, B. (2008), "Data Revisions are not Well-Behaved," Journal of Money, Credit and Banking, 40, 319-340.

Aruoba, S.B. and F.X. Diebold (2010), "Real-Time Macroeconomic Monitoring: Real Activity, Inflation, and Interactions," American Economic Review, 100, 20-24.

Aruoba, S.B., F.X. Diebold, J. Nalewaik, F. Schorfheide, and D. Song (2011), "Improving GDP Measurement: A Measurement Error Perspective," Manuscript in progress, University of Maryland, University of Pennsylvania and Federal Reserve Board.

Bates, J.M. and C.W.J. Granger (1969), "The Combination of Forecasts," Operations Research Quarterly, 20, 451-468.

Bloom, N., M. Floetotto, and N. Jaimovich (2009), "Really Uncertain Business Cycles," Manuscript, Stanford University.

Carter, C.K. and R. Kohn (1994), "On Gibbs Sampling for State Space Models," Biometrika, $81,541-553$.

Diebold, F.X. and J.A. Lopez (1996), "Forecast Evaluation and Combination," In G.S. Maddala and C.R. Rao (eds.) Handbook of Statistics (Statistical Methods in Finance), North- Holland, 241-268.

Faust, J., J.H. Rogers, and J.H. Wright (2005), "News and Noise in G-7 GDP Announcements," Journal of Money, Credit and Banking, 37, 403-417.

Fixler, D.J. and J.J. Nalewaik (2009), "News, Noise, and Estimates of the "True" Unobserved State of the Economy," Manuscript, Bureau of Labor Statistics and Federal Reserve Board.

Hamilton, J.D. (1989), "A New Approach to the Economic Analysis of Nonstationary Time Series and the Business Cycle," Econometrica, 57, 357-384.

Kim, C.-J. and C.R. Nelson (1999a), "Has the U.S. Economy Become More Stable? A Bayesian Approach Based on a Markov-Switching Model of the Business Cycle," Review of Economics and Statistics, 81, 608-616.

Kim, C.-J. and C.R. Nelson (1999b), State Space Models with Regime Switching, MIT Press. 
Kishor, N.K. and E.F. Koenig (2011), "VAR Estimation and Forecasting When Data are Subject to Revision," Journal of Business and Economic Statistics, in press.

Mankiw, N.G., D.E. Runkle, and M.D. Shapiro (1984), "Are Preliminary Announcements of the Money Stock Rational Forecasts?" Journal of Monetary Economics, 14, 15-27.

Mankiw, N.G. and M.D. Shapiro (1986), "News or Noise: An Analysis of GNP Revisions," Survey of Current Business, May, 20-25.

McConnell, M. and G. Perez-Quiros (2000), "Output Fluctuations in the United States: What Has Changed Since the Early 1980s?" American Economic Review, 90, 1464-1476.

Nalewaik, J.J. (2010), "The Income- and Expenditure-Side Estimates of U.S. Output Growth," Brookings Papers on Economic Activity, 1, 71-127 (with discussion).

Nalewaik, J.J. (2011), "Estimating Probabilities of Recession in Real Time Using GDP and GDI," Journal of Money, Credit and Banking, in press.

Schorfheide, F. (2005), "Learning and Monetary Policy Shifts," Review of Economic Dynamics, 8, 392-419.

Statistisches Bundesamt, Wiesbaden (2009), "National Accounts: Gross Domestic Product in Germany in Accordance with ESA 1995 - Methods and Sources," Subject Matter Series, 18.

Stock, J.H. and M.W. Watson (2002), "Has the Business Cycle Changed and Why?" In M. Gertler and K. Rogoff (eds.), NBER Macroeconomics Annual, Cambridge, Mass.: MIT Press, 159-218.

Timmermann, A. (2006), "Forecast Combinations," In G. Elliot, C.W.J. Granger and A. Timmermann (eds.), Handbook of Economic Forecasting, North-Holland, 136-196. 